\title{
vivienda unifamiliar REEUWIJK
}

HEIN SALOMONSON, arquitecto

$161 \cdot 113$

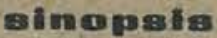

Situada en una zona tacustre. tipicaménte holandesa, ta casa ha sido cohistraida formando qu grac, patio, enn objeto de

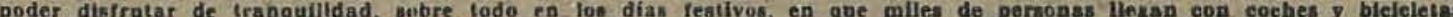
procedentes de ta cercana oludad de Rutterdam, en busea de espareimiento.

Su planta aparece claramente divlaida en 'trea $20 n a s$. Is 8 . alberga la sala de estar - en conexión sblerta con ta coclnaan vestuario y un sseo con duchs-para bañiatas-, y un sótano. La B. alofa trę dormitorios y el bafio. Bn la eons N. proyectads on principio como trastero, se ha hablitiado una pequeúa habitacion-eatudio.

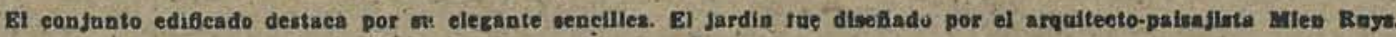

Este ediflcio se construyó como ucasa para vacaciones», si bien se ha acondicionado adecuada. mente para poder ser habitada en cualquier época del año. Ocupada por un ingeniero consultor y su familia, constituye al mismo tiempo un centro de reunión para el y sus colaboradores y un sitio apropiado para llevar a cabo un tipo de trabajo - como es el suyo-que exige concentración.

Está situada en un distrito lacustre, típicamente holandés, donde existen carreteras estrechas y puentes levadizos que salvan los canales.

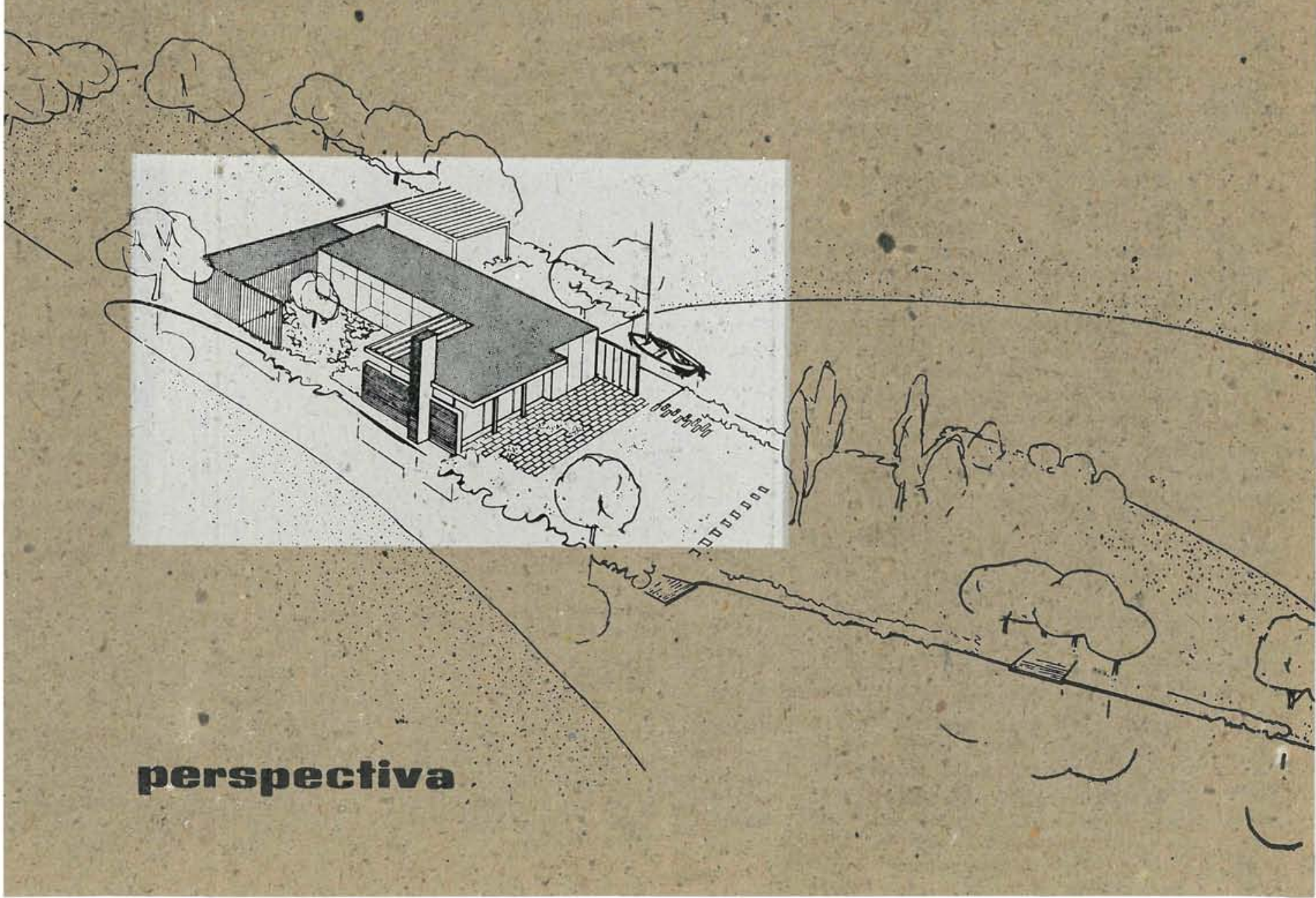




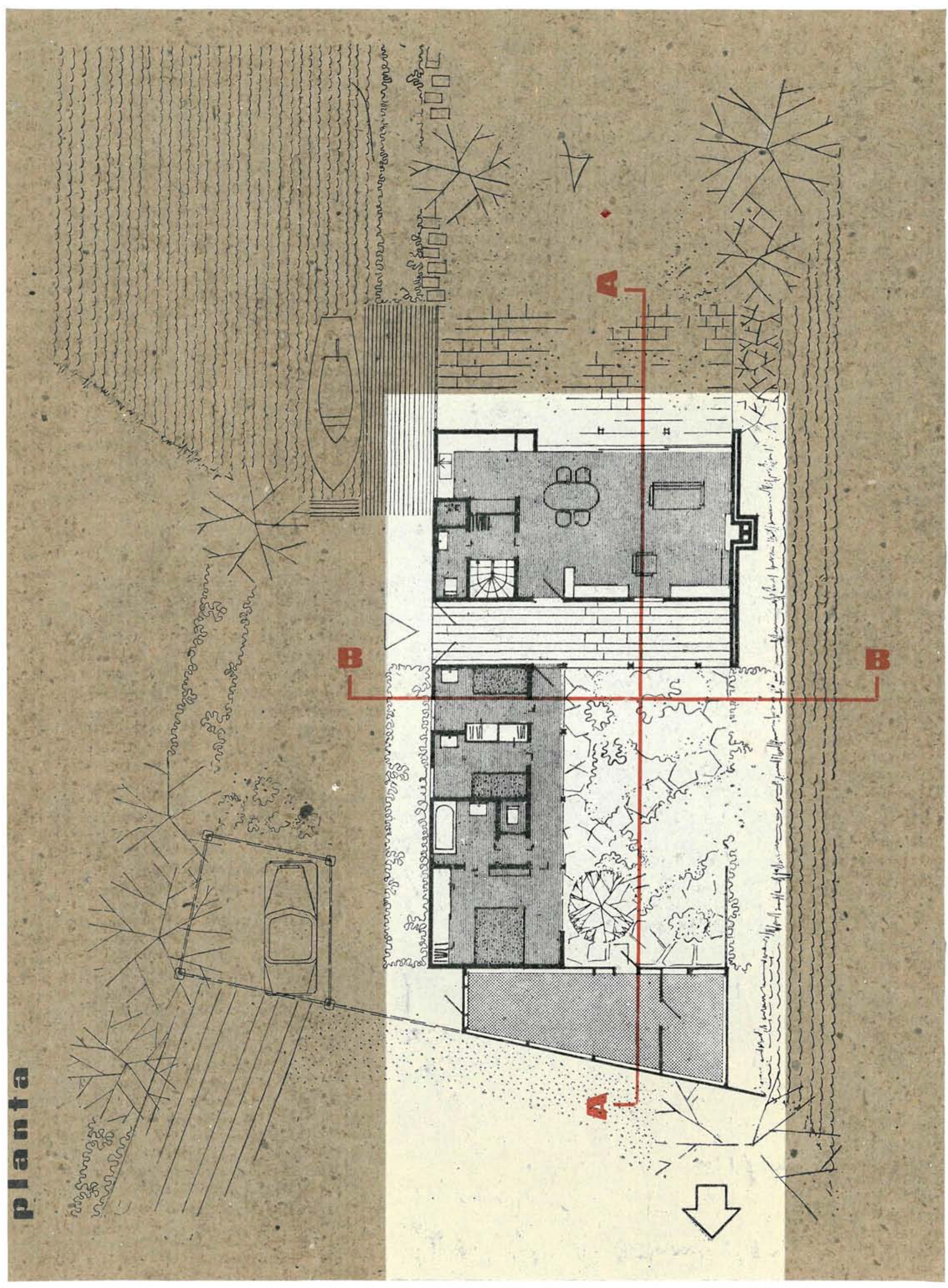




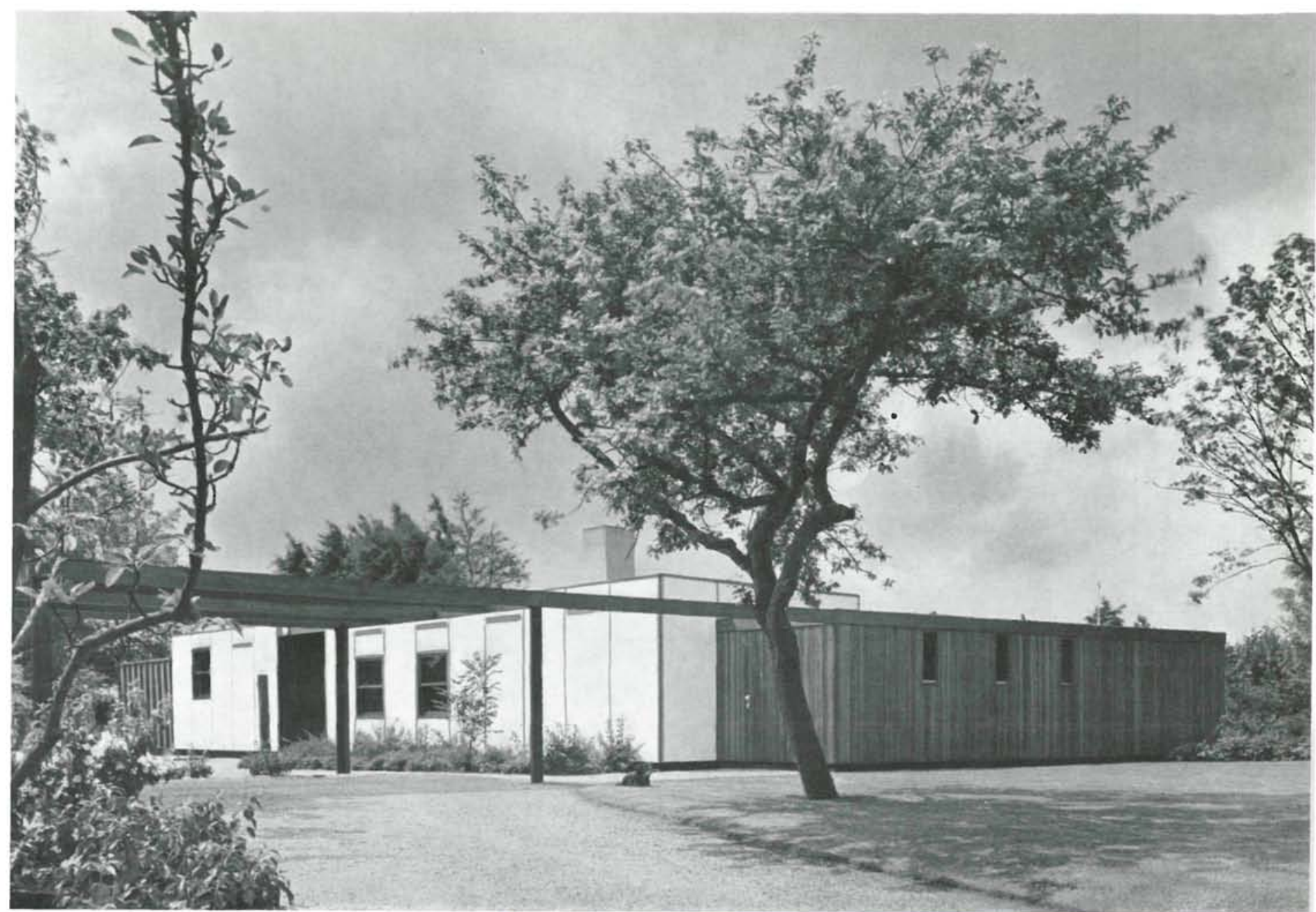

En los domingos y días festivos llegan, desde la cercana ciudad de Rotterdam, miles de personas en coches y bicicletas buscando esparcimiento, por cuya razón la casa ha sido construida formando un gran patio, en el cual pueden disfrutar del descanso apetecido en los días más bulliciosos.

La planta está organizada en tres zonas: la sur, con la sala de estar-en conexión abierta con la cocina-, un vestuario y un aseo con ducha para los bañistas, y un sótanc. La este contiene tres dormitorios y el baño. En la zona norte - proyectada para trastero en un principio-, se habilitó una pequeña habitación-estudio.
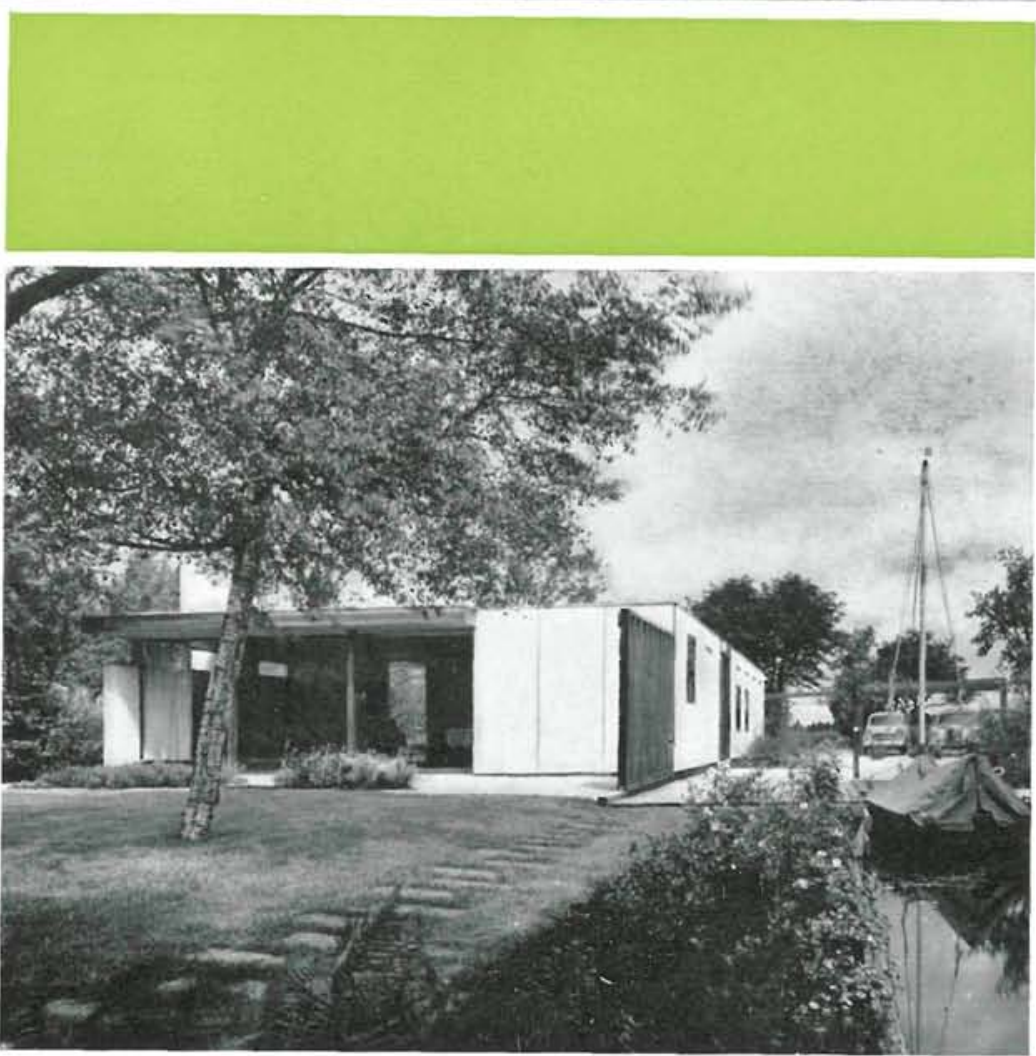

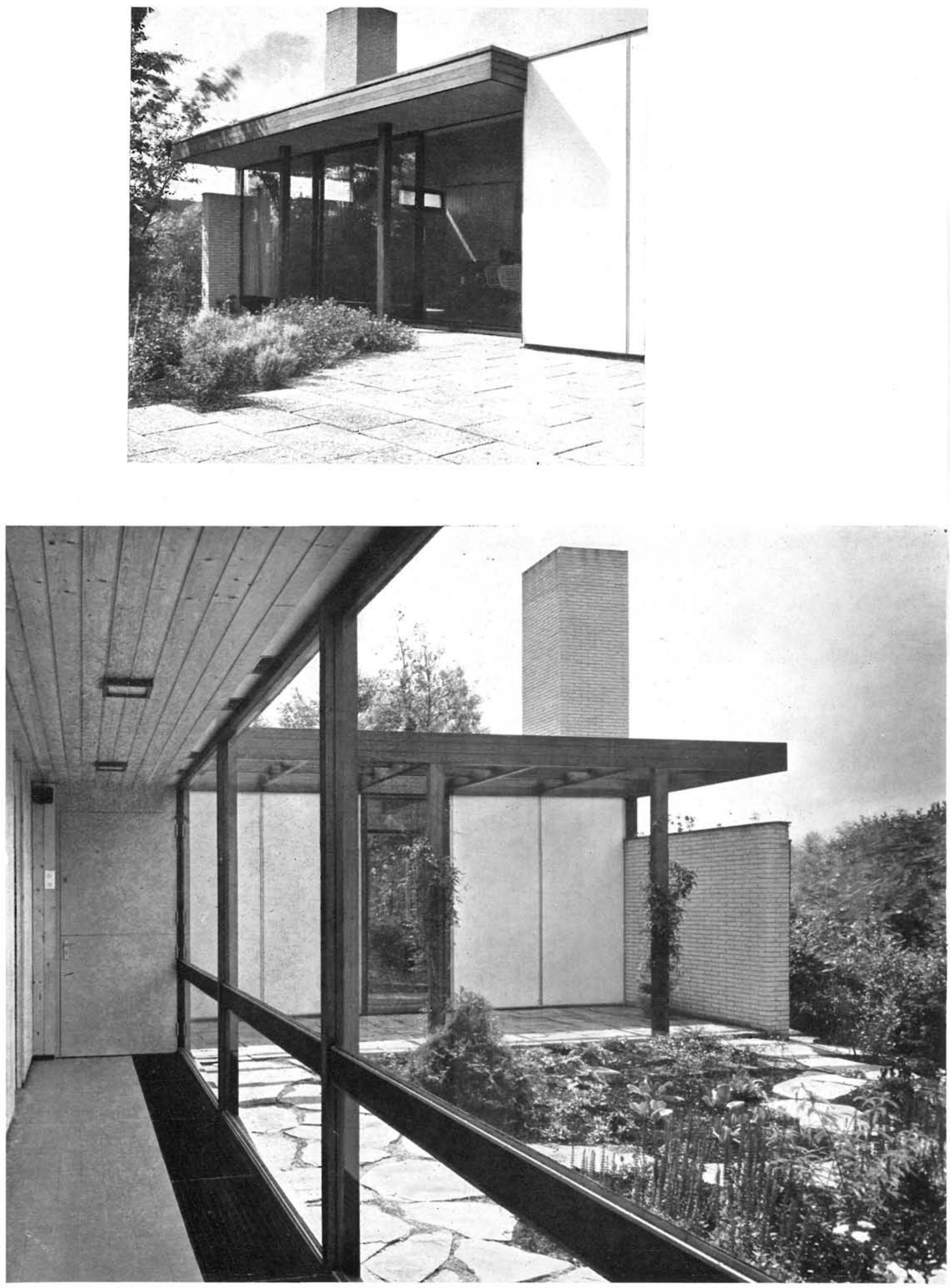


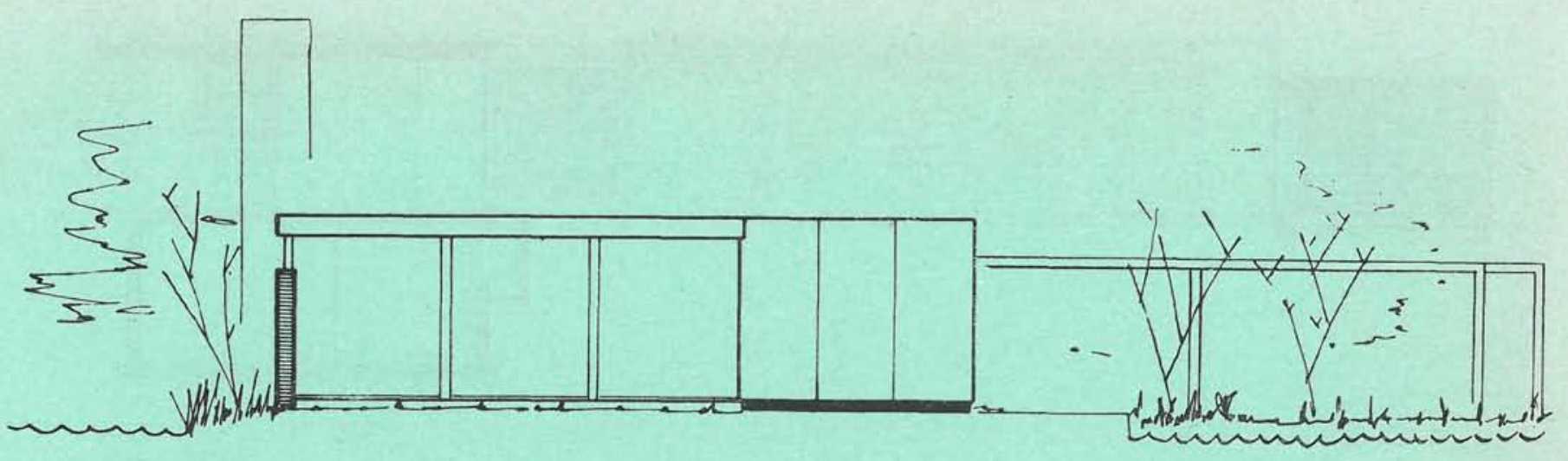

ตอยำำ

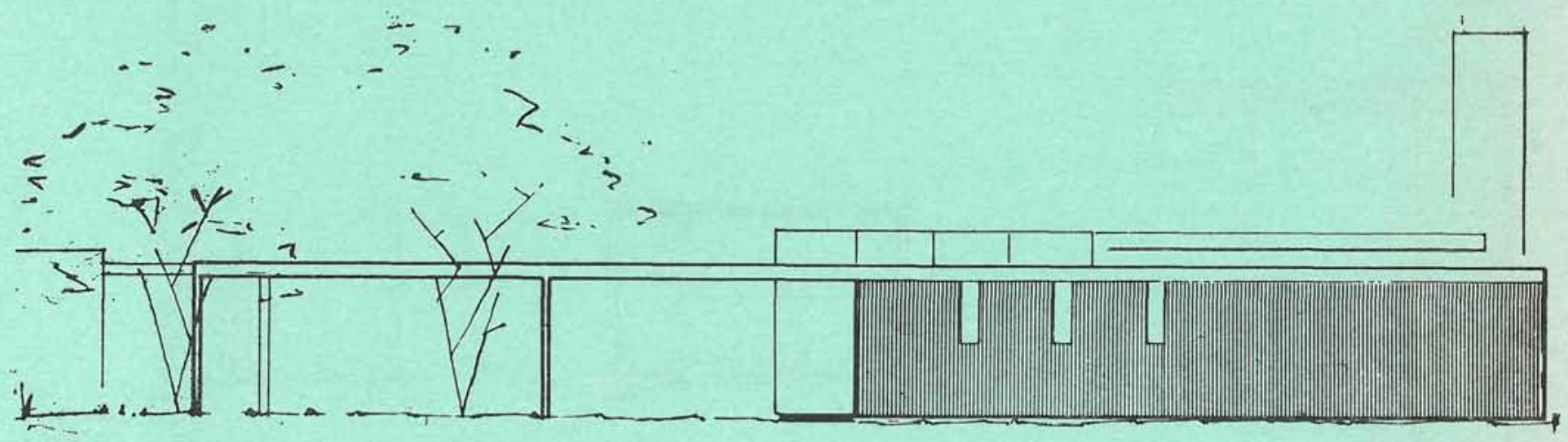

Scer

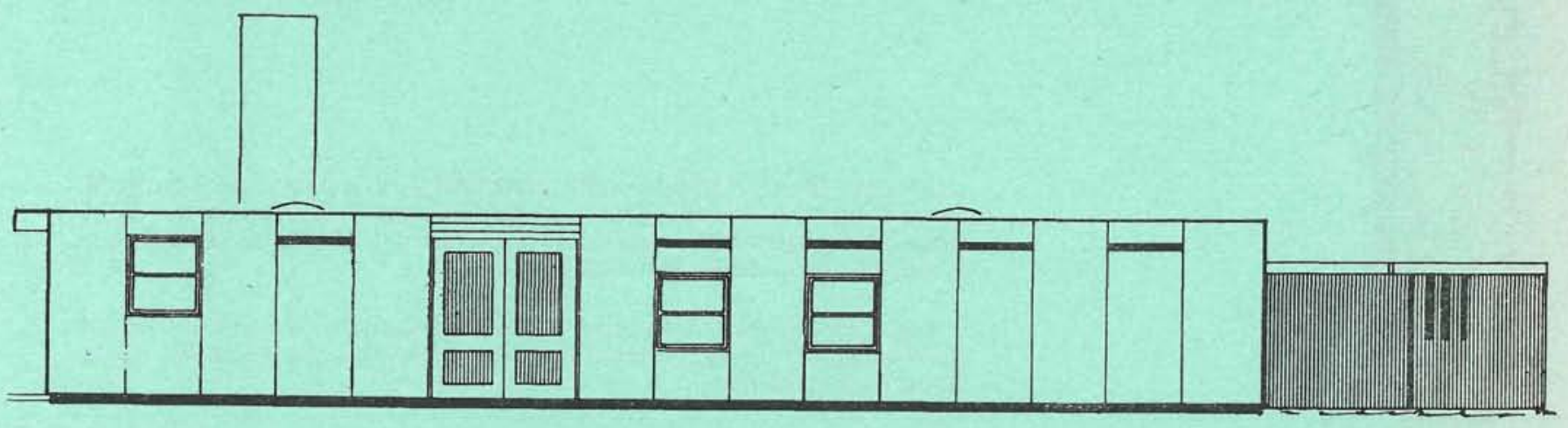

Es혼룰

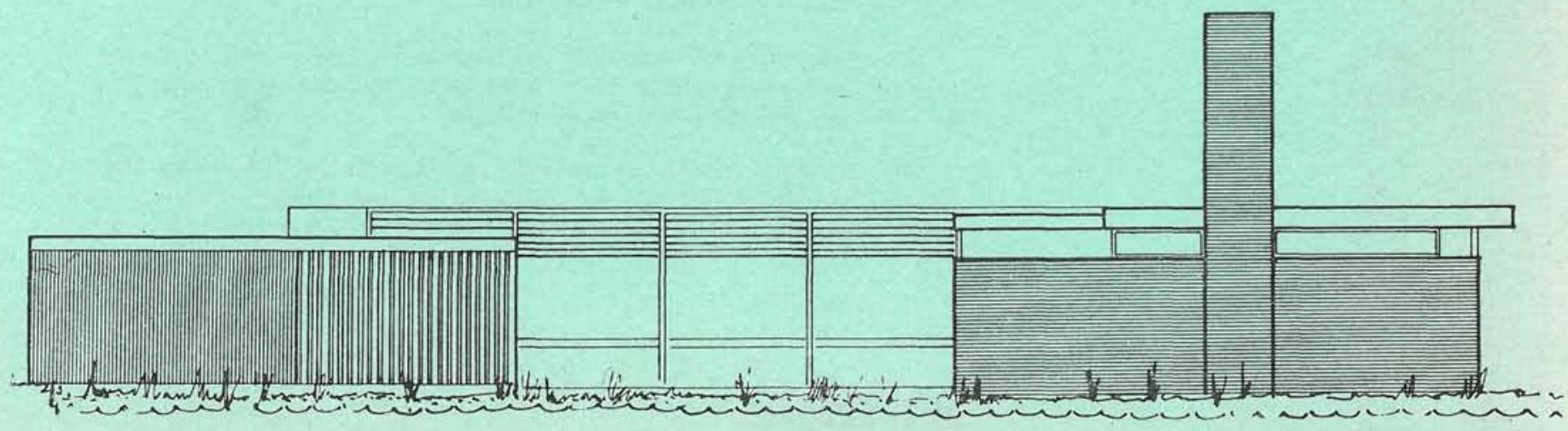
Dessere 

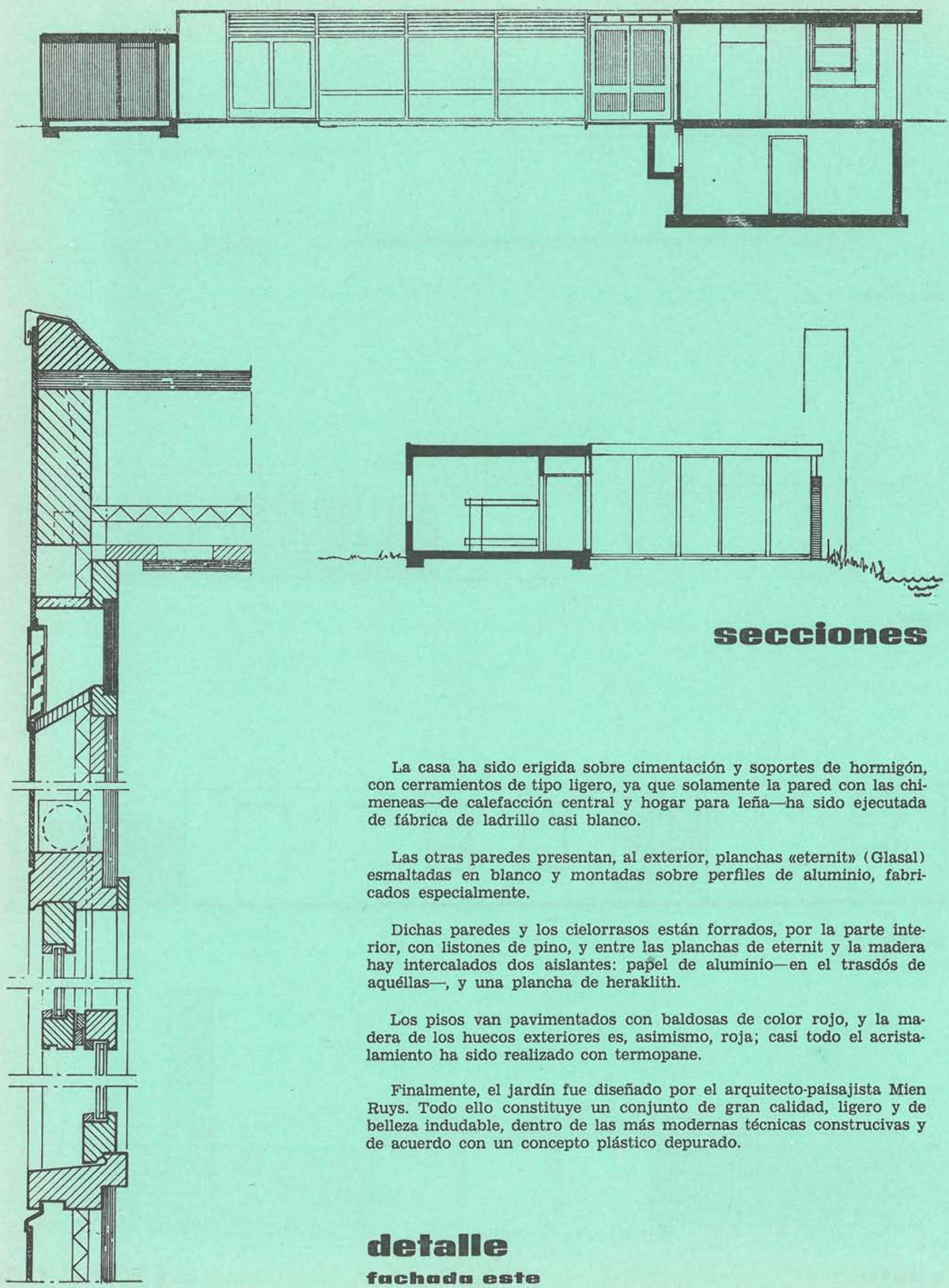

Seceiomes

La casa ha sido erigida sobre cimentación y soportes de hormigón, con cerramientos de tipo ligero, ya que solamente la pared con las chimeneas-de calefacción central y hogar para leña-ha sido ejecutada de fábrica de ladrillo casi blanco.

Las otras paredes presentan, al exterior, planchas «eternit» (Glasal) esmaltadas en blanco y montadas sobre perfiles de aluminio, fabricados especialmente.

Dichas paredes y los cielorrasos están forrados, por la parte interior, con listones de pino, y entre las planchas de eternit y la madera hay intercalados dos aislantes: papel de aluminio-en el trasdós de aquéllas-, y una plancha de heraklith.

Los pisos van pavimentados con baldosas de color rojo, y la madera de los huecos exteriores es, asimismo, roja; casi todo el acristalamiento ha sido realizado con termopane.

Finalmente, el jardín fue diseñado por el arquitecto-paisajista Mien Ruys. Todo ello constituye un conjunto de gran calidad, ligero y de belleza indudable, dentro de las más modernas técnicas construcivas y de acuerdo con un concepto plástico depurado.

\section{detalle}

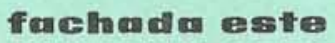



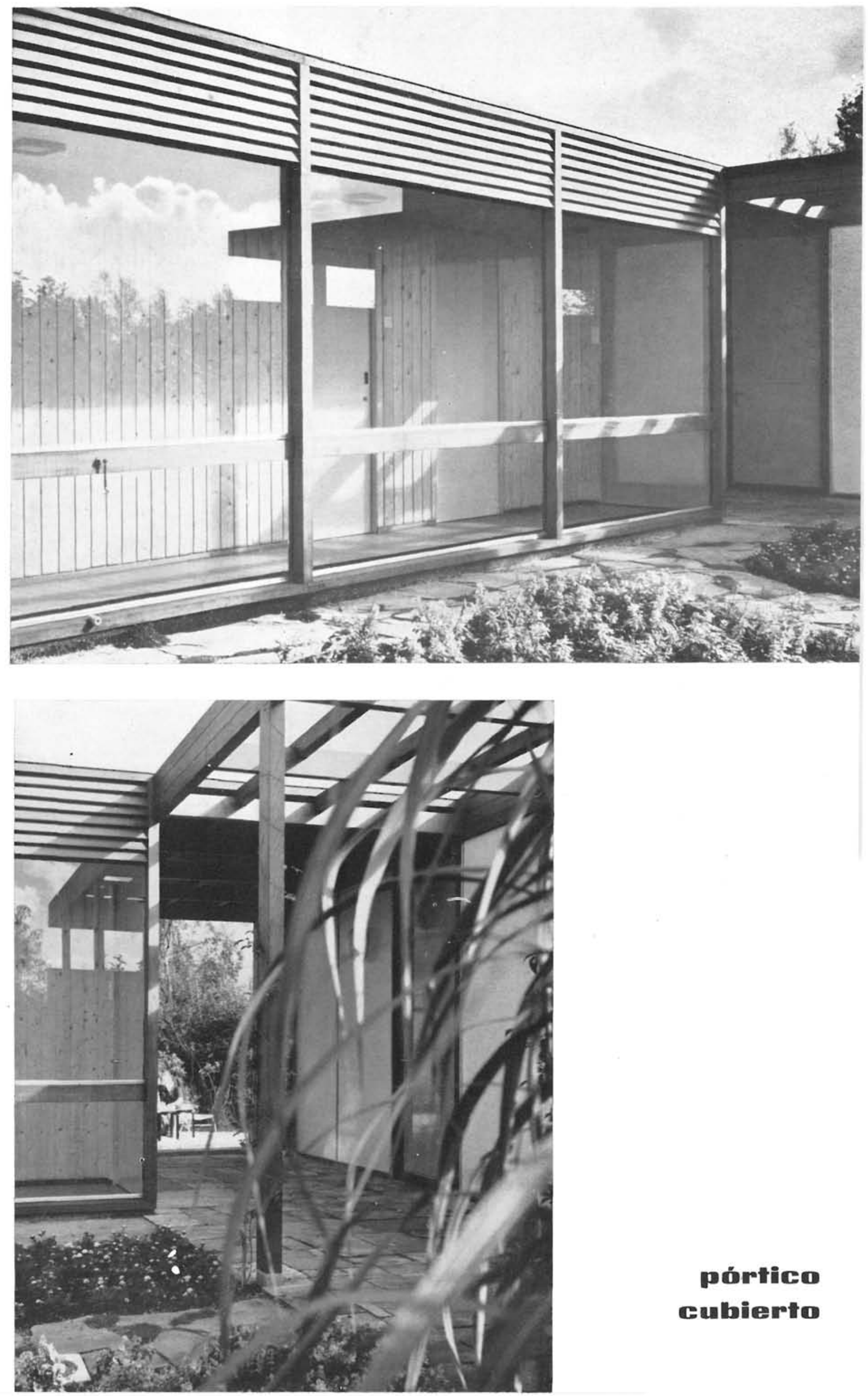

\author{
pórtico
}

Cubierto 

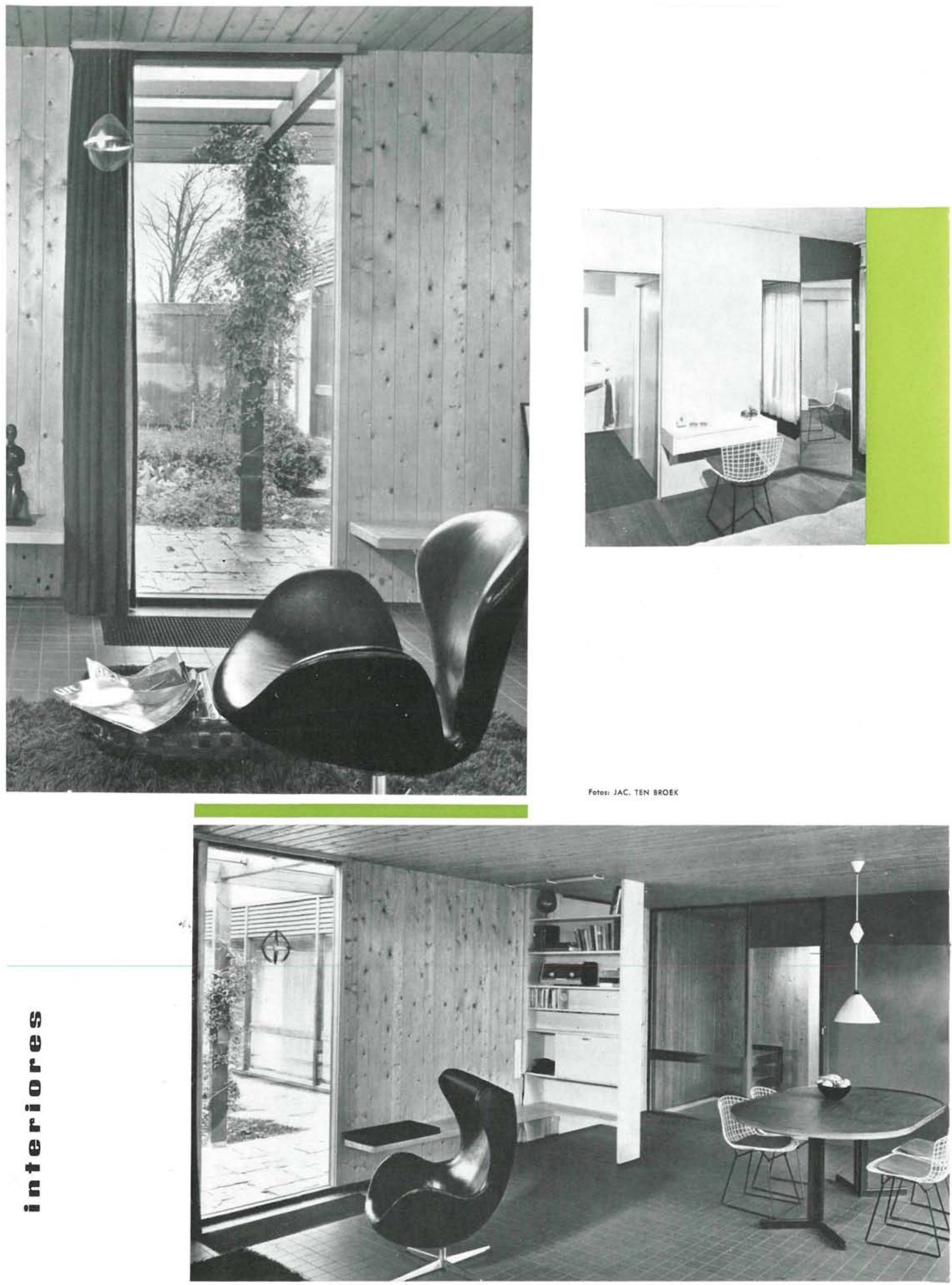


\section{Maisom Reeuwijk}

Hein Salomonson, architecte.

Située dans une zone lacustre, typiquement hollandaise, cette maison a été conçue pour former un jardin intérieur, afin de pouvoir jouir de tranquillité, surtout les jours fériés, quand des milliers de personnes, en quête de détente, arrivent en voitures et à bicyclettes de la cité de Rotterdam, toute proche.

Le plan de cette maison apparaît clairement divisé en trois zones: au sud, la salle de séjour-en connexion ouverte avec la cuisine-un vestiaire et une toilette-douche, pour les baigneurs, et un sous-sol. A l'est, trois chambres et la salle de bains. Au nord, projetée en principe comme grenier, a été aménagée une petite chambre-studio.

L'ensemble est remarquable pour son élégante simplicité. Le jardin a été dessiné par l'architecte-paysagiste Mien Ruys.

\section{"Reeumijlk" lhousse}

Hein Salomonson, architect.

This house is situated in typical Dutch scenery; very flat country, interspersed with lakes. The building encloses in the centre a large patio, so that the occupants can enjoy privacy and quiet on Sundays and other holidays, when a large number of holidaymakers come to the district by car and motorbike from the nearby city of Rotterdam.

The plan distribution is clearly divided into three zones. The southern one includes the living room, directly connected with the kitchen, a dressing room, a wash and changing room (for swimmers) and a basement. The eastern one is taken up with three bedrooms and a bathroom. The northern part, which initially was meant to be a spare room, is now used as a study.

The project, as a whole is outstanding for its elegant simplicity. The garden was designed by the landscape architect Mien Ruys.

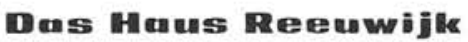

Hein Salomonson, Architekt.

Das Haus Reeuwijk liegt in einer typisch holländischen Seengegend, und wurde mit einem grossen Innenhof gebaut, um den Bewohnern die Möglichkeit zu geben, die Feiertage in Ruhe zu geniessen, denn gerade dann kommen tausende von Menschen auf der Suche nach Weite mit Autos und Fahrrädern aus dem nahegelegenden Rotterdamm gefahren.

Der Grundriss des Hauses lässt deutlich drei Teile erkennen:

Der südliche Teil beherbergt: Aufenthaltsraum mit offener Verbindung zur Küche, Umkleideraum, Dusch- und Waschräume für Badegäste und Keller. Der Ostteil besteht aus drei Schlafräumen und Bad. Der Nordteil, der ursprünglich als Abstellraum geplant war, wurde als Studierzimmer hergerichtet.

Der ganze Bau hebt sich durch seine elegante Einfachheit hervor. Der Garten wurde vom Gartenbauarchitekten Mien Ruys angelegt. 